\title{
Uso de antidepressivos e ansiolíticos entre estudantes do curso de farmácia em uma instituição privada e uma pública do interior da Bahia
}

\author{
Use of antidepressants and anxiolytics among pharmacy course students in a private and public \\ institution in the interior of Bahia \\ Uso de antidepresivos y ansiolíticos en estudiantes del curso de farmacia de una institución pública \\ y privada del interior de Bahia
}

Recebido: 14/06/2021 | Revisado: 23/06/2021 | Aceito: 28/06/2021 | Publicado: 12/07/2021

\author{
Mickaelly Stefanie Paes Souza \\ ORCID: https://orcid.org/0000-0003-1929-4283 \\ Faculdade Independente do Nordeste, Brasil \\ E-mail: Mickassouza02@gmail.com \\ Rebeca Laís Matos de Lima Almeida \\ ORCID: https://orcid.org/0000-0002-5748-1437 \\ Faculdade Independente do Nordeste, Brasil \\ E-mail: rebecalaismatos@outlook.com \\ Aline Teixeira Amorim \\ ORCID: https://orcid.org/0000-0003-2597-8665 \\ Faculdade Independente do Nordeste, Brasil \\ E-mail: aline.amorim2011@ hotmail.com \\ Tayanne Andrade dos Santos \\ ORCID: https://orcid.org/0000-0002-7977-6308 \\ Faculdade Independente do Nordeste, Brasil \\ E-mail: tayanne.as@hotmail.com
}

\begin{abstract}
Resumo
A depressão e a ansiedade são doenças que vêm acometendo cada vez mais jovens por todo o mundo. É nessa fase que também são registrados o maior percentual de risco ao suicídio. Como forma de tratamento para os transtornos psíquicos, a farmacoterapia é bastante utilizada, mas, com ela, surgem várias questões e discussões sobre o uso de psicoativos por jovens, dentre as quais destacam-se a automedicação. Por meio de um questionário semiestruturado, este estudo analisou a quantidade de universitários de uma instituição privada e uma pública do interior da Bahia, que foram diagnosticados com depressão e/ou ansiedade. Os resultados mostraram uma prevalência de jovens com idades entre 24 e 28 anos (50\%) e revelou 77,7\% dos entrevistados iniciou o uso de medicamentos após o ingresso no ensino superior. Observou-se ainda que as mulheres compunham a maior parte dos estudantes diagnosticados com ansiedade ou depressão, dado que pode ser associado a aspectos sócio-histórico-culturais.
\end{abstract}

Palavras-chave: Ansiedade; Depressão; Farmacoterapia; Universitários; Ensino superior.

\begin{abstract}
Depression and anxiety are diseases that are afflicting more and more young people all over the world. It is at this stage that the highest percentage of risk for suicide is also recorded. As a form of treatment for psychic disorders is used pharmacotherapy, but there are several questions and discussions about the use of psychoactive drugs by young people because of self-medication. This study used a questionnaire and analyzed the number of university students from a private and a public institution in the interior of Bahia, that were diagnosed with depression or anxiety. The results showed a prevalence of young people aged between 24 and 28 years $(50 \%)$ and revealed that $77.7 \%$ of the interviewees started using medications after entering higher education. It was also observed that women were the majority of students diagnosed with anxiety or depression. The reason for this may be historical and cultural aspects. Keywords: Anxiety; Depression; Pharmacotherapy; College students; University education.
\end{abstract}

\section{Resumen}

La depresión y la ansiedad son enfermedades que afectan cada vez a más jóvenes en todo el mundo. Es en esta etapa donde también se registra el mayor porcentaje de riesgo de suicidio. Como forma de tratamiento de los trastornos mentales, la farmacoterapia es muy utilizada, pero con ella surgen varias preguntas y discusiones sobre el uso de psicofármacos por parte de los jóvenes, entre las que destaca la automedicación. A través de un cuestionario semiestructurado, este estudio analizó el número de estudiantes universitarios de una institución privada y pública del interior de Bahía, que fueron diagnosticados con depresión o ansiedad. Los resultados mostraron una prevalencia de 
jóvenes de entre 24 y 28 años (50\%) y revelaron que el 77,7\% de los encuestados comenzaron a usar medicamentos después de ingresar a la educación superior. También se observó que las mujeres constituyeron la mayoría de los estudiantes diagnosticados de ansiedad o depresión, ya que puede asociarse con aspectos socio-histórico-culturales.

Palabras clave: Ansiedad; Depresión; Farmacoterapia; Estudiantes universitarios; Enseñanza superior.

\section{Introdução}

A transição da adolescência para a fase adulta é marcada por profundas transformações na vida do indivíduo. Essas mudanças repentinas costumam despertar estranhamentos tanto nos jovens quanto nos adultos, podendo causar ansiedade depressiva, baixa autoestima e sentimentos de anulação (Biazus \& Ramires, 2012).

Definida como uma disfunção que acontece nos neurotransmissores, a depressão pode ter influências de herança genética ou até mesmo falhas em algumas áreas cerebrais decorrentes de mudanças psicoemocionais ao longo da vida do indivíduo. Quando causada por problemas psicológicos e/ou ambientais, a depressão é tida como resultado de fatores exógenos (Moreira et al., 2014).

Indivíduos que apresentam quadro depressivo podem ser acometidos também pelo transtorno de ansiedade, que é caracterizado pela presença de preocupação excessiva, inquietação, dificuldade de concentração e insônia (Zuardi, 2017). Há um predomínio mundial no diagnóstico de transtornos psiquiátricos relacionados à ansiedade e à depressão, com 3,6\% apresentando ansiedade e 4,4\% com depressão (World Health Organization, 2017 como citado em Bauchrowitz, Paz, Müller, Possagno, \& Minozzo, 2019).

Nesse contexto, o tratamento medicamentoso surge como recomendação para alguns pacientes, que não raramente associam a medicação a tabus, preconceitos e ao medo de ser rotulado. Como consequência dessa visão estereotipada, a automedicação é adotada como alternativa em muitos casos. É nesse momento que se torna fundamental o acompanhamento de profissionais habilitados e da família, para que os instruam sobre os benefícios esperados do tratamento medicamentoso e riscos potenciais (Moreira et al., 2014).

Durante o seu processo de adaptação ao ensino superior, o indivíduo enfrenta um período de amadurecimento, passando da fase da adolescência para as responsabilidades da vida adulta, tornando-se, assim, um adulto jovem (Lopez et al., 2011). Essas mudanças podem causar conflitos psicológicos advindos das dificuldades de aprendizagem, das cobranças acadêmicas e de adaptação a um novo ritmo de vida (Borine, Wnaderley, \& Bassitt, 2015). Outros fatores que também influenciam no surgimento desses transtornos são as tentativas de conciliar estudo e trabalho, o que pode expor o estudante a situações contínuas de estresse. Por conseguinte, podem ser desenvolvidos transtornos de ansiedade e de depressão. Dados apontam que o jovem que está na vida acadêmica tem maior probabilidade de desencadear quadros depressivos, sendo que de $15 \%$ a $29 \%$ apresentam transtornos psiquiátricos ao longo da sua vida estudantil e $28 \%$ da população com idade entre 18 e 24 anos possuem sintomas depressivos (Cybulski \& Mansani, 2017).

Com a pandemia pela Covid-19, uma das medidas de segurança adotadas foi o isolamento social, comportamento que tem afetado negativamente a qualidade de vida da população, sobretudo no que tange à saúde mental. Concomitantemente, tem-se observado um agravamento dos distúrbios psicológicos pela dificuldade de equilíbrio psíquico e emocional, sobre os quais alguns tratamentos como a psicoterapia e a farmacoterapia auxiliam na recuperação dos indivíduos. Entretanto, é preciso cautela quanto à prescrição de antidepressivos com avaliação do risco e benefício, pois o uso desses medicamentos não deve ser compreendido como principal ferramenta terapêutica, principalmente em casos leves (Liu et al., 2020; Damasceno, Santana, Santos, Souza, \& Santos, 2019).

Tendo um maior conhecimento sobre o crescimento desordenado da depressão no final da adolescência e início da fase adulta, é necessário que ela seja diagnosticada e tratada o mais breve, com medidas farmacológicas ou não farmacológicas. Como medidas farmacológicas são utilizados os medicamentos classificados como psicotrópicos. Esses 
medicamentos são constituídos por substâncias que atuam diretamente no Sistema Nervoso Central (SNC) (Avelar et al., 2019). O uso desses fármacos também pode trazer alguns efeitos colaterais e interações medicamentosas que podem ser prejudiciais à saúde dos usuários.

Sendo assim, esta pesquisa consiste em uma análise do uso de antidepressivos e ansiolíticos entre jovens universitários, identificando se houve surgimento ou acentuação de transtornos psíquicos quando relacionados aos estudos, trabalho e quadro pandêmico. É feita ainda uma abordagem da necessidade de reajuste de dose e adesão aos medicamentos em situação de uso, bem como os possíveis danos causados pela falta da terapêutica adequada e da automedicação.

O índice de pessoas que são diagnosticadas com depressão e ansiedade vem crescendo progressivamente, principalmente quando se trata de jovens. Outro aspecto em relação à rede causal do problema é o reduzido número de atividades voltadas à prevenção da depressão. Sendo que, existem diversos dispositivos sociais, como escolas, Centro de Referência em Assistência Social (CRAS) e serviços de saúde que podem ser ambientes multiplicadores de atividades sobre a ansiedade e a depressão. Soma-se a isso, a ausência de programas de esclarecimentos à população sobre o transtorno, o preconceito da família, o não reconhecimento da pessoa do seu real problema e a não adesão ao tratamento medicamentoso e psicoterápico. Por isso, este trabalho tem como problemática a necessidade de determinar o quantitativo da população universitária que possui as patologias citadas em instituições de ensino superior do interior da Bahia.

A utilização de medicamentos antidepressivos e ansiolíticos foi considerada como a principal e mais efetiva estratégia para o tratamento de pacientes que são diagnosticados com esses transtornos, quando comparada com as psicoterapias. A hipótese do presente trabalho é que diante do cenário atual da pandemia da COVID-19 há uma elevada utilização de ansiolíticos e antidepressivos entre estudantes do curso de farmácia de instituições pública e privada.

Os medicamentos antidepressivos e ansiolíticos estão entre as principais estratégias de tratamento para pacientes que são diagnosticados com depressão e ansiedade. Embora existam outros métodos, como o tratamento não farmacológico, que é comprovadamente eficaz, o uso de psicotrópicos sobressai aos demais cuidados que são prescritos por médicos devido à grande variedade de efeitos terapêuticos. Estes, por sua vez, tendem a aliviar os sintomas do indivíduo por meio dos seus potenciais ansiolíticos, antidepressivos, sedativos, dentre outros.

O aumento dos casos de depressão e ansiedade em jovens universitários são evidências concretas desse cenário de saúde mental, podendo ser caracterizado como o mais novo mal da contemporaneidade. Neste contexto, os estudos e pesquisas que estão relacionadas à medicalização de antidepressivos e ansiolíticos e suas formas de tratamento e cuidado à saúde é de extrema importância, pois discutem sobre estratégias de prevenção e de qualidade de vida de universitários que são diagnosticados com tais transtornos.

Nessa perspectiva, a presente pesquisa teve como público-alvo estudantes do curso de farmácia de uma instituição de ensino privada e uma pública do interior da Bahia, e propôs-se a analisar a quantidade de alunos que possui depressão e/ou ansiedade, que fazem uso de medicamentos para este fim e que recorrem à automedicação. Discutir os fatores que favorecem esses transtornos e abordar as possíveis consequências de um tratamento sem acompanhamento médico também foram focos deste estudo.

\section{Metodologia}

A pesquisa decorreu em caráter exploratório, quantitativo e qualitativo. Um estudo qualitativo propõe-se a discutir conceitos a partir de dados empíricos; o método quantitativo confere maior exatidão ao modo como uma variável funciona e se relaciona com as outras (Koche, 2011). A pesquisa descritiva analisa e compara fatos sem manipulá-los, sempre com o intuito de descobrir com a maior perfeição possível, como um fenômeno ocorre e sua regularidade, procurando entender as diversas relações que do comportamento humano, tanto de forma isolada quanto em grupos mais complexos (Manzato \& Santos, 2011). 
A pesquisa exploratória tem como objetivo oferecer ao pesquisador uma maior intimidade com o estudo em questão. Geralmente é utilizado em casos nos quais é necessário que o problema seja definido, a fim de obter dados e, finalmente, desenvolver uma abordagem. Esse tipo de estudo tem a finalidade de explorar uma situação, de modo a compreendê-la com o uso de métodos bastante vastos. Tais métodos podem partir de levantamentos em fontes secundárias como as documentais, por exemplo, levantamentos de experiência e estudos de casos selecionados (Vieria, 2002).

A amostra foi composta por um total de 350 alunos do curso de Farmácia de uma instituição privada e uma pública do interior da Bahia. Os dados foram coletados por via remota, através de um questionário feito pelas pesquisadoras. Os questionários avaliados consideraram pessoas que utilizam medicamentos antidepressivos e/ou ansiolíticos, com o intuito de ter uma melhor representatividade no que se refere à parcela selecionada do universo pesquisado.

A pesquisa ocorreu por via remota, por meio de um questionário aplicado para alunos do curso de Farmácia em uma instituição de ensino privado e uma pública no interior da Bahia.

O instrumento utilizado para a obtenção de dados um questionário (Apêndice A), elaborado de forma online, via Google Forms, e aplicado remotamente pelo Whatsapp pelo e-mail.

Foi enviado um link para o participante que continha o Termo de Consentimento Livre e Esclarecido. Após ler e aceitar participar da pesquisa, o entrevistado recebia o questionário contendo perguntas objetivas e subjetivas com foco nos objetivos do presente estudo.

Os dados obtidos a partir dos questionários foram transcritos para a plataforma Excel versão 2010 Microsoft $®$ e exportado para o software SPSS. Com a finalidade de organizar e obter um resumo conciso dos dados, utilizou-se a estatística descritiva como análise de frequências, cálculos de medidas de tendência central como média, mediana e moda, e de medidas de dispersão como variância e desvio padrão. Também foram realizadas análises inferenciais, utilizando teste Qui-Quadrado para variáveis qualitativas. Para variáveis quantitativas foram utilizados o teste t ou Mann-Whitney, a partir do qual os grupos foram comparados com a adoção de um nível de significância de 5\%.

Foram incluídos na pesquisa estudantes maiores de 18 anos e que estavam com matrículas ativas no curso de Farmácia de uma instituição pública e de uma instituição privada em um município do interior da Bahia.

Foram excluídos da presente amostra os participantes menores de 18 anos, aqueles que não aceitaram participar da pesquisa ou os cujos questionários respondidos estavam incompletos.

A pesquisa foi submetida e aprovada pelo Comitê de Ética e Pesquisa da Faculdade Independente do Nordeste FAINOR, Parecer número 4.613.110, e conduzida de acordo com a Resolução N. º 466/2012 da Comissão Nacional de Saúde. Essa autorização permite a realização de pesquisa envolvendo seres humanos, por ocasião da coleta de dados.

\section{Resultados e Discussão}

Foram entrevistadas 45 pessoas, das quais $73,3 \%$ são do sexo feminino e $26,7 \%$ do sexo masculino. Em um estudo realizado por Damasceno et al. (2019) sobre os riscos do uso de antidepressivos entre jovens universitários da área da saúde, a maioria dos participantes também eram mulheres, com percentual de 79,2\%. Valor semelhante foi encontrado por Mesquita, Lemes e Carrijo (2016) ao analisar a depressão entre estudantes de cursos da área da saúde de uma universidade em Mato Grosso, que teve $71 \%$ de mulheres dentre os estudantes entrevistados. Para a análise das idades dos participantes, foram consideradas três faixas etárias, conforme a Tabela 1. 
Tabela 1. Faixa etária dos entrevistados.

\begin{tabular}{ccc}
\hline Idade & Frequência & Porcentagem \\
\hline $19-23$ & 4 & $26,7 \%$ \\
$24-28$ & 9 & $60,1 \%$ \\
$29-50$ & 2 & $13,4 \%$ \\
Tota1 & 15 & $100,0 \%$ \\
\hline
\end{tabular}

Fonte: Autores

A frequência do número de entrevistados em relação à idade (ver Tabela 1) revelaram que 26,7\% têm entre 19 e 23 anos; $60,1 \%$ possuem idades entre 24 e 28 anos e 13,4\% têm entre 29 e 50 anos.

Quanto ao estado civil, 50\% das pessoas disseram serem solteiras e $16 \%$ serem casadas ou divorciadas, um percentual de 34\% não respondeu à questão. Das 5 pessoas $(17,2 \%)$ que têm filhos, $60 \%$ são casadas e $40 \%$ são solteiras ou divorciadas. Da mesma forma, no estudo de Mesquita et al. (2016), a maioria dos participantes eram solteiros (83\%). Esse fato revela um aumento da incidência de mulheres no âmbito acadêmico e um adiamento da idade de oficialização nupcial (Ribeiro, 2009 como citado em Orlando, 2017). Nessa perspectiva, na pesquisa feita por Vieira e Alves (2014), no ano de 2010 a idade média de mulheres e homens em união formal era, respectivamente, 31,2 e 33,5 anos.

Outro dado de relevância foi que 53,3\% dos entrevistados eram estudantes do curso de farmácia. Destes, $75 \%$ estudavam em instituição privada de ensino superior e $25 \%$ em instituição pública. Além disso, daqueles que possuíam diagnóstico para ansiedade e/ou depressão e faziam uso de medicamento, 62,5\% faziam parte de instituição privada.

Quanto à presença de ansiedade e depressão, 65\% dos entrevistados afirmaram terem sido diagnosticados com algum desses transtornos. Uma taxa igualmente elevada foi obtida no estudo de Damasceno et al. (2019), que teve um percentual de $79,1 \%$ de usuários de medicamentos antidepressivos.

Das 18 pessoas que fazem uso de medicamentos, $10(55,5 \%)$ responderam que não trabalham e apenas estudam.

Considerando o total de pessoas que usam psicotrópicos, $27,7 \%$ associam o uso ao trabalho e 77,7\% iniciaram o uso após o ingresso no ensino superior. Dos que fazem uso mediante orientação profissional, 81,2\% recebeu prescrição médica e 6,25\% fazem uso por recomendação de familiares ou amigos, como mostra a Tabela 2. Esses percentuais apresentam equivalência com os resultados do estudo de Damasceno et al. (2019), no qual 90,7\% dos entrevistados receberam orientação médica para o uso de psicoativos e 7\% afirmou fazer uso de medicamentos por conta própria ou por recomendação de familiares.

Tabela 2. Distribuição de frequência dos profissionais que orientaram o uso de medicamentos psicoativos.

\begin{tabular}{ccc}
\hline Quem orientou & Frequência & Porcentagem \\
\hline Médico apenas & 12 & $80,0 \%$ \\
Médico e farmacêutico & 1 & $6,7 \%$ \\
Apenas Farmacêutico & 1 & $6,7 \%$ \\
Me informei por conta & 1 & $6,7 \%$ \\
própria & 15 & $100,0 \%$ \\
Total & 15
\end{tabular}

Fonte: Autores. 
A Tabela 3 expõe que 43,75\% dos universitários ajustaram a dose durante a pandemia pela Covid-19. Esse resultado pode ser associado ao crescimento de problemas que envolvem a saúde mental, em tempos de pandemia, conforme sugere um estudo de Moura, Carvalho, Resende, Silva e Grillo (2021) sobre o uso de substâncias e fatores de proteção em período de pandemia pelo coronavírus. Os autores afirmam que, durante a quarentena, o número de casos de ansiedade e estresse chegaram a aumentar $80 \%$.

Tabela 3. Ajuste de dose durante a pandemia pela Covid-19.

\begin{tabular}{ccc}
\hline Ajuste de dose & Frequência & Porcentagem \\
\hline Ajustou a dose & 7 & $43,75 \%$ \\
Não ajustou a dose & 9 & $56,25 \%$ \\
Total & 16 & $100 \%$ \\
\hline
\end{tabular}

Fonte: Autores.

Outra informação relevante referiu-se ao uso de medicamentos psicoativos, pelos universitários, desde o início da pandemia pela Covid-19. Os resultados mostraram que um número considerável de estudantes (43,75\%) teve a dose ajustada no período, contudo, 81,25\% não interrompeu o tratamento durante a pandemia. Em consonância com esses dados, o Conselho Federal de Farmácia (CFF) (2020) registrou um aumento na venda de medicamentos psiquiátricos durante a pandemia. No caso dos antidepressivos e estabilizadores de humor, houve um aumento de 13,84\% de vendas no ano de 2020, se comparado ao ano anterior. $\mathrm{O}$ mesmo conselho atribui esse índice às mudanças que a pandemia provocou na vida das pessoas, tais como $\mathrm{o}$ confinamento, o isolamento social, o desemprego e a crise financeira (CFF, 2020).

A Tabela 4 mostra que $68,75 \%$ dos entrevistados já interromperam o tratamento sem recomendação médica. De acordo com o estudo de Marinho, Nascimento e Nicoletti (2019), a interrupção do uso de medicamentos por conta própria pode ser explicada pelas dúvidas dos entrevistados quanto ao tratamento e ao abandono deste. Em uma pesquisa sobre o uso de ansiolíticos, Fávero (2017) associa a interrupção sem acompanhamento médico como fator potencializador de sintomas de abstinência, como nervosismo, insônia, náuseas, agitação e pânico.

Tabela 4. Interrupção do tratamento sem orientação médica.

\begin{tabular}{ccc}
\hline Interrupção do tratamento & Frequência & Porcentagem \\
\hline Sim & 11 & $68,75 \%$ \\
Não & 5 & $31,25 \%$ \\
Total & 16 & $100 \%$ \\
\hline
\end{tabular}

Fonte: Autores.

Conforme apontado por Ribeiro, Cruz, Marchi, Tirapelli e Miasso (2014), no Brasil, os sintomas depressivos estão presentes entre $15 \%$ a $25 \%$ dos estudantes universitários durante o período de formação acadêmica. Esse dado foi ratificado nesta pesquisa, que teve $77,7 \%$ dos participantes registrando o início do uso de psicofármacos após começarem a cursar a graduação.

Ainda segundo a pesquisa de Ribeiro et al. (2014), o consumo de antidepressivos foi predominante em estudantes do 
sexo feminino, constatação igualmente feita neste estudo. Essa informação, para Mesquita et al. (2016) reforça a histórica associação da figura feminina à sensibilidade e zelo. Pode-se ainda relacionar esse dado à maior predisposição da mulher em procurar ajuda profissional e, consequentemente, ter o diagnóstico de ansiedade e depressão.

Acerca da prevalência da referida faixa etária neste e em outros estudos, Cybulski e Mansani (2017) alertam que além de provocar sofrimento psíquico, a depressão pode prejudicar o desempenho acadêmico do indivíduo e suas relações sociais como um todo. $\mathrm{O}$ fato da maioria dos participantes estarem matriculados em universidades particulares pode ser associado à grande variedade desse tipo de instituição na cidade em que a pesquisa foi desenvolvida.

Para Damasceno et al. (2019), o uso majoritário de medicamentos pelos universitários que possuem diagnóstico para ansiedade ou depressão apontam para a necessidade um olhar mais criterioso quanto à prescrição de psicoativos, pois podem se tornar o tratamento principal e não um auxílio terapêutico para os transtornos psíquicos de ansiedade e depressão.

\section{Considerações Finais}

O estudo desenvolvido com os estudantes universitários mostrou que, de fato, a maioria dos jovens diagnosticados com ansiedade ou depressão faz uso de medicamentos como estratégia de tratamento, sendo que 78\% iniciaram o uso após o ingresso no ensino superior. Além disso, foi observado uma mudança no padrão de consumo de ansiolíticos e antidepressivos durante a pandemia pela Covid-19, pois $43,75 \%$ dos usuários afirmaram terem feito reajuste de dose nesse período e mais de $80 \%$ dos jovens analisados não interromperam o uso ao longo desse tempo. Isso revela que a tensão e a incerteza de um cenário de adoecimento da população têm provocado impactos psicoemocionais significativos na saúde mental das pessoas. Portanto, a realização de mais estudos que tratem da relação entre estresse estudantil e crescimento de transtornos de ansiedade e depressão tende a ser um instrumento significativo para a análise de intervenções e de tratamentos eficazes no que tange a saúde mental.

\section{Referências}

Avelar, L. P., Castilho, L. S., Abreu, M. H., Vilaça, E. L., Resende, V. L., \& Silva, M. E. (2019). A prescrição de medicação psicotrópica e o conhecimento da portaria regulatória brasileira por cirurgiões-dentistas. Cadernos Saúde Coletiva, 27(3), 338-344. https://www.scielo.br/j/cadsc/a/p7WyJYmqd yVQd5MpHmfHVZp/?lang=pt\&format=pdf. 10.1590/1414-462X201900030382

Bauchrowitz, C., Paz, L. E., Muller, E. V., \& Halila, G. C. (2019). Prevalência de uso de psicofármacos por acadêmicos: efeitos do processo de graduação. Brazilian Journal of Development, 5(11), 24915-24933. https://www.researchgate.net/publication/337987802 _Prevalencia_de_uso_de_psicofar macos_por_academicos_efeitos_do_processo_de_graduacao. 10.34117/bjdv5n11-170

Biazus, C. B., \& Ramires, V. R. (2012). Depressão na adolescência: uma problemática dos vínculos. Psicologia em Estudo, 17(1), 83-91. https://www.scielo.br/j/pe/a/RSkXKnYD4frXYKQrmzGdGMn/?lang=pt

Borine, R. C., Wanderley, K. S., \& Bassit, D. P. (2015). Relação entre qualidade de vida e o estresse em acadêmicos da área da saúde. Estudos interdisciplinares em Psicologia, 6(1), 100-118. http://pepsic.bvsalud.org/pdf/eip/v6n1/a08.pdf. 10.5433/2236-6407

Conselho Federal de Farmácia. (2020). Venda de medicamentos psiquiátricos cresce na pandemia. Brasil: Autor. http://covid19.cff.org.br/venda-demedicamentos-psiquiatricos-cresce-na-pandemia/

Cybulsk, C. A., \& Mansani, F. P. (2017). Análise da Depressão, dos Fatores de Risco para Sintomas Depressivos e do Uso de Antidepressivos entre Acadêmicos do Curso de Medicina da Universidade Estadual de Ponta Grossa. Revista Brasileira de Educação Médica, 41(1), 92-101. https://www.scielo.br/j/rbem/a/qZQbVnkyfT9pPQP3qqSPQHw/?lang=pt\&format=pdf. http://dx.doi.org/10.1590/1981-52712015v41n1RB20160034

Damasceno, E. M., Santana, B. M., Santos, L. G., Souza, M. F., \& Santos, E. R. (2019). Riscos do uso de antidepressivos entre jovens universitários da área da saúde. Revista Saúde Viva Multidisciplinar da AJES, 2(2), 13-24. http://www.revista.ajes.edu.br/revistas-noroeste/index.php/revisajes/article/view/11

Fávero, V. R., Sato, M. O., \& Santiago, R. M. (2017). Uso de ansiolíticos: abuso ou necessidade? Visão Acadêmica, 18(4), 98-106. https://revistas.ufpr.br/academica/article/view/57820/34821. http://dx.doi.org/10.5380/acd.v18i4.57820

Koche, J. C. (2011). Fundamentos de metodologia científica. Vozes.

Liu, S., Yang, L., Zhang, C., Xiang, Y., Liu, Z., \& Hu, S. (2020). Online mental health services in China during the COVID-19 outbreak. The Lancet Psychiatry, 7(4), e17-e18. http://europepmc.org/article/MED/32085841. 10.1016/S2215-0366(20)30077-8 
Research, Society and Development, v. 10, n. 8, e29610817177. 2021

(CC BY 4.0) | ISSN 2525-3409 | DOI: http://dx.doi.org/10.33448/rsd-v10i8.17177

Lopez, M. R., Ribeiro, J. P., Ores, L. C., Jansen, K., Souza, L. D., Pinheiro, R. T., \& Silva, R. A. (2011). Depressão e qualidade de vida em jovens de 18 a 24 anos no sul do Brasil. Revista de Psiquiatria do Rio Grande do Sul, 33(2), 103-108. https://www.scielo.br/j/rprs/a/HjPQny Gq9c4p8qsMxWCTnrS/?lang=pt\&format=pdf

Manzato, A. J., \& Santos, A. B. (2012). A elaboração de questionários na pesquisa quantitativa. Departamento de Ciência de Computação e Estatística IBILCE - UNESP. http://www.inf.ufsc.br/ vera.carmo/Ensino_2012_1/ELABORACAO_QUESTIONARIOS_PESQUISA_QUANTITATIVA.pdf

Marinho, T. N., Nascimento, L. M., \& Nicoletti, C. D. (2019). Depressão entre universitários: revisão integrativa dos medicamentos antidepressivos mais utilizados entre os acadêmicos de universidades no Brasil. Semioses: Inovação, Desenvolvimento e Sustentabilidade, 13(4), 15-33. https://revistas.unisuam.edu.br/index.php/semioses/article/view/489. https://doi.org/10.15202/1981996x.2019v13n4p15

Mesquita, A. M., Lemes, A. G., Carrijo, M. V., Moura, A. A., Couto, D. S., Rocha, E. M., \& Volpato, R. J. (2016). Depressão entre estudantes de cursos da área da saúde de uma universidade em Mato Grosso. Journal Health NPEPS, 1(2), 218-230. https://periodicos.unemat.br/index.php/j hnpeps/article/view/1433/1503

MOREIRA, M. S., Morais, R. G., Moreira, E. A., Leite, S. F., Teixeira, C. C., Silva, M. E., \& Freitas, D. F. (2014). Uso de psicofármacos em crianças e adolescentes. Revista da Universidade Vale do Rio Verde, 12(2), 1013-1049. http://periodicos.unincor.br/index.php/revistaunincor/article/view/1821. http://dx.doi.org/10.5892/ruvrd.v12i2.1821

Moura, C. S., Carvalho, D. F., Resende, C. M., Silva, I. C., \& Grillo, M. H. (2021). O uso de álcool e outras drogas e seus possíveis fatores de proteção em tempos de pandemia. Revista Episteme Transversalis, 12(1), 135-154. http://revista.ugb.edu.br/ojs302/index.php/episteme/article/view/2373

Orlando, C. A. K. (2017). Seletividade marital no Brasil entre 1995, 2005 e 2015 (Trabalho de conclusão de curso). Universidade Federal do Rio de Janeiro.

Ribeiro, A. G., Cruz, L. P. Marchi, K. C., Tirapeli, C. R., \& Miasso, A. I. (2014). Antidepressivos: uso, adesão e conhecimento entre estudantes de medicina. Ciência \& Saúde Coletiva, 19(6), 1825-1833. https://www.scielo.br/j/csc/a/f9cfdWDrzv3tpvLDmRhDz3t/abstract/?lang=pt. https://doi.org/10.1590/1413-81232014196.06332013

Vieira, J. M., \& Alves, L. C. (2014). Comportamento da nupcialidade no Brasil e nas unidades federativas em 2000 e 2010. Anais do XIX Encontro Nacional de Estudos Populacionais, São Pedro, SP, Brasil, 19.

Vieria, V. A. (2002). As tipologias, variações e características da pesquisa de marketing. Revista da FAE, 5(1), 61-70. file://C:/Users/Valqu\%C3\%ADria/Downloads/449-1180-1-SM.pdf

Zuardi, A. W. (2017). Características básicas do transtorno de ansiedade generalizada. Medicina (Ribeirão Preto), 50(supl.1), 51-55. https://www.revistas.usp.br/rmrp/article/view/127538. https://doi.org/10.11606/issn.2176-7262.v50isup11.p51-55 